\title{
Alargamiento mandibular mediante tracción en pacientes con síndrome de Pierre Robin
}

\author{
C. Gloria Morovic I. '; Luis Monasterio A.'
}

\section{Treatment of Pierre Robin syndrome by gradual mandibular distraction}

\begin{abstract}
Bone lengthening by gradual oseaus traction has become a gooc tool to solve skeielal malformation of the lace. This melhod was used in wo parients o: Pierre Robin syndrome wilh upper airwoy obstruction by severs mandibular hypoplasia. Fallowing mandibular traction a signiticant increase 116 lo 22 mml in the dimensions of the mandible was obtained, thus allowing trcchecstomy tube removal in the first cose and making mechanical ventilatory support no further necessary in the second patient. This method of Ireatment mov provide salisfactory functional and aesthetic correclion lo severely affected Pierre Robin syndrome patienls with minimal morbidily.
\end{abstract}

(Key words: Pierre Robin syndrome, bone lengthening, bone gradual distraction, mandible, remodeling.)

El alargamiento de un hueso mediante tracción gradual del callo óseo fue descrito en $1905^{1}$ con el propósito de corregir discrepancias de ambas extremidades inferiores y temporalmente abandonado por las complicaciones que causaba, como necrosis de la piel, edema, desviación y falta de consolidación de los segmentos. En 1950, en Rusia, se recogió la idea, encontrando que si se mantenía el periostio los resultados eran muy superiores, cambiando la osteotomía por corticotomía 2,3 .

En 1973 se describieron importantes alargamientos obtenidos aplicando el mismo principio en mandíbulas de perro. Después de varios estudios experimentales, se han desarrollado programas de tracción en pacientes pediátricos con hipoplasia mandibular, mediante un aparato extraoral, logrando sorprendentes resultados. Simultáneamente en México se han acumulado numerosos casos de excelentes resultados utilizando tracción mandibular unilateral, bilateral, sea en una $o$ dos direcciones ${ }^{48}$.

Se describen los resultados de la tracción

I. Cirugía Plástica Pedriátrica, Hospital Luis Calvo Mackena y Clínica Alemana. en dos pacientes con sindrome de Pierre Robin y obstrucción respiratoria relacionada con la severidad de su hipoplasia mandibular uno de los cuales requería traqueostomía y el otro apoyo permanente con ventilación mecánica.

\section{Material y Método}

Desde el comienzo de nuestro programa de tracción mandibular progresiva, en noviembre 1994, se han presentado dos casos de síndrome de Picre Robin en crítica situación respiratoria, en los cuales se realizó tracción mandibular bilateral unidireccional

En ambos casos, bajo anestesia general nasotraqueal, se realizaron corticotomías externas en los ángulos de la mandfbula, manteniendo indemnidad de la cortical intema. A conlinuación se colocaron clavos de fijación en ambos cabos de las corticotomias, en los que se instaló el tractor ("distractor") externo tipo Molina (diseñado por Fernando Molina, México, y fabricado por Wells Johnson Co.). Cinco Jias después se inició tracción ósea dando un giro de $360^{\circ}$ diariamemte al tornillo, para producir una separación de $1 \mathrm{~mm}$ entre ambos segmentos oseos, 10 que continus realizandose durante 20 a 25 días, para, posteriormente, mantener una distancia fija -mientras se consolidaba el callo óseo- por aproximadamente 6 a 8 semanas. Finalizado este periodo, se verificó la formación de buen callo óseo, lo que permitió retirar el aparato. Se realizó registro folográfico y radiológico antes, durante y después de terminado el procedimiento, para registrar su progreso. 


\section{Resultados}

Caso 1. Varón de 8 meses de cdad con labio leporino bilateral y síndrome de Pierre Robin con gran hipoplasia mandibular $y$ obstrueción respiratoria que hicicron necesario mantenerle con tracueostomía dexde las primeras semanas de vida. Se colocaron dos distractores mandibulares unidireccionitles, obteniéndose alargamientos de $1,8 \mathrm{~cm}$ a litdo derecho y $2.2 \mathrm{~cm}$ en el izquierdo, con lo cual desapareció la obstrucción resp1ratoria y fue nosible cerrar la traqueostomía. Despucs de 6 semanas de fijación se registró formación de un buen callo oseo y se retiraron los draclores (ligura 1 ).
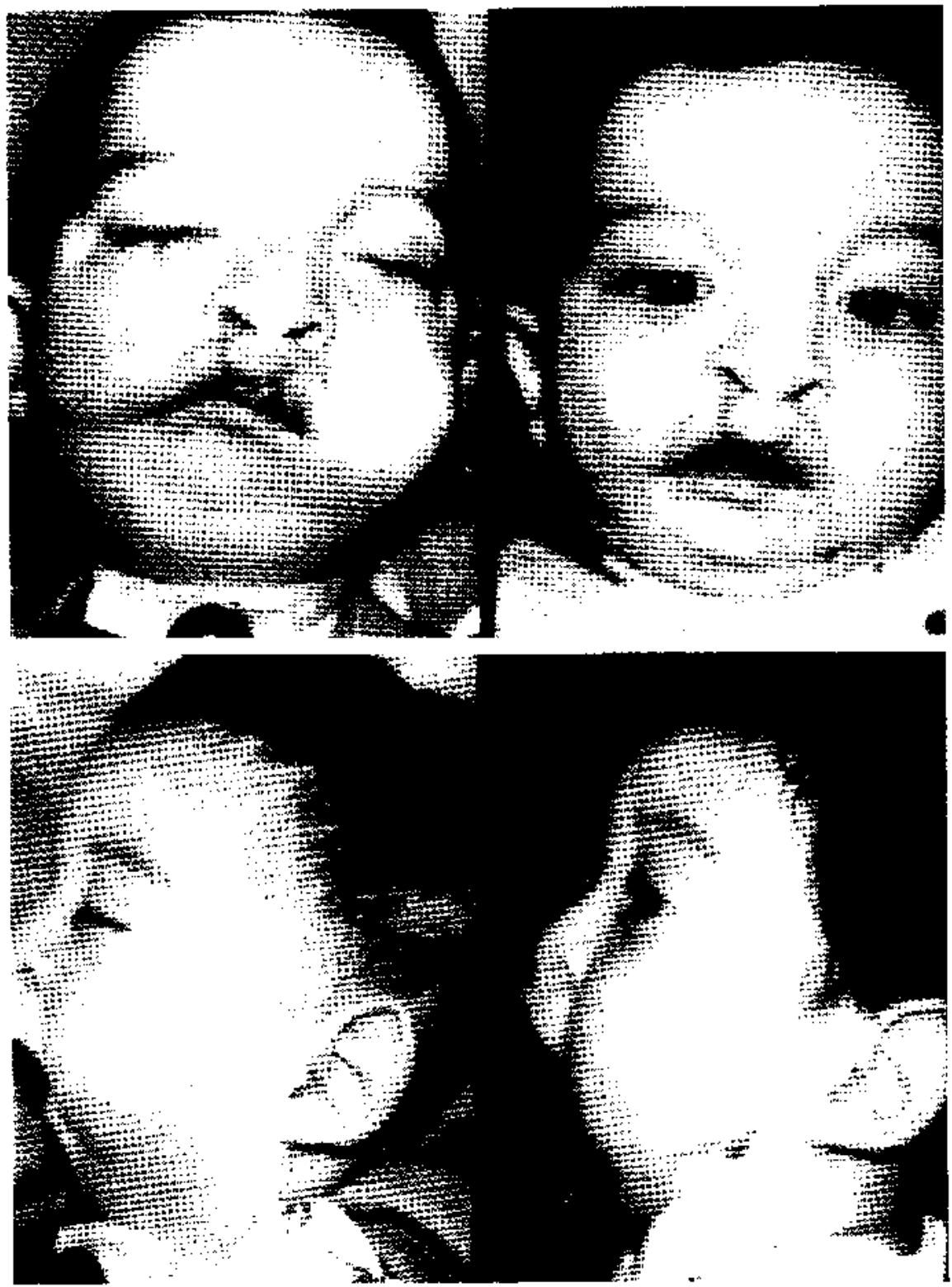

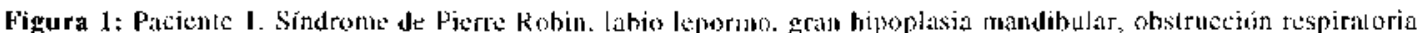
traqueostombía. Arriba: asperto frortal antes (izqujesda y después (derecha) de la traceión gradual mandibułas. Abaju: aspecto laterd muestra con mayor mtidez la grill hipoplasin mindibular. antes (izquierda) y despulse (derecha) de la traceión ósea gradual de la mandíbula. 
Caso 2. Varón de 1 mes de edad, sindrome de Pierre Robin con gran hipoplasia mandibular e intensa obstrucción respiratoria secundaria debido a la cual fue preciso mantenerle en una unidad de cuidados intensiros concctado a un ventilador mecánico. Sufrió paro respiratorio cada vez que sc intentó desconectarle de la asistencia respiraloria. Aunque no se encontró en la literatura información sobrc la aplicación del procedimicnto a tan temprana edad, se decidió realizar tracción mandibular bilateral, unidireccional, manteniéndole en ventilador mecánico, con to que se obtuvo un alargamicnto del cuerpo mandibular de $1 \mathrm{~cm}$ al lado derecho y de $1,6 \mathrm{~cm}$ en el izquierdo, que permitio retirarle el ventilador antes del lérmino del proceso activo de tracción. si bien a causa de un episodio de reflujo gastroesolágico masivo y bronconeumonía grave fue neccsario reentubarlo temporalmente. Luego de completat 5 semanas de fijación, se retiró el tractor mandibular, evolucionando sin obstrucción respiratoria (figura 2 ).

El período de seguimiento del primer paciente es de 9 meses hasta la fecha de envío a publicación. En ambos niños la tracción ósea se realizó en forma ambulatoria con control semanal y con ella se obtuvo un avance considerable en las dimensiones de la mandíbula. Como complicación, la extrusión de clavo ocurrió en tres oporlunidades, obligando a reinstalar el aparato. lo que, sin embargo, no alteró la progresión del desarrollo.

\section{Comentario}

En el manejo de estos pacientes con gran hipoplasia mandibular y en situación crítica respiratoria sc han cmpleado varias técnicas quirúrgicas, desde la fijación traumática de la lengua, pasando por el despegamiento muscular del piso de la boca ${ }^{9}$ y otras que tendían a aliviar transitoriamente los efectos de la anomalía. La tracción ósca cs un procedimiento relativamente simple, que abre un nuevo horizomte a los niños con malformaciones severas del esqueleto facial, dando una solución definitiva al problema, la que, además, facilita la expansión de lodos los tejidos blandos involucrados con excelente resultado estético, funcional y mínima morbilidad.
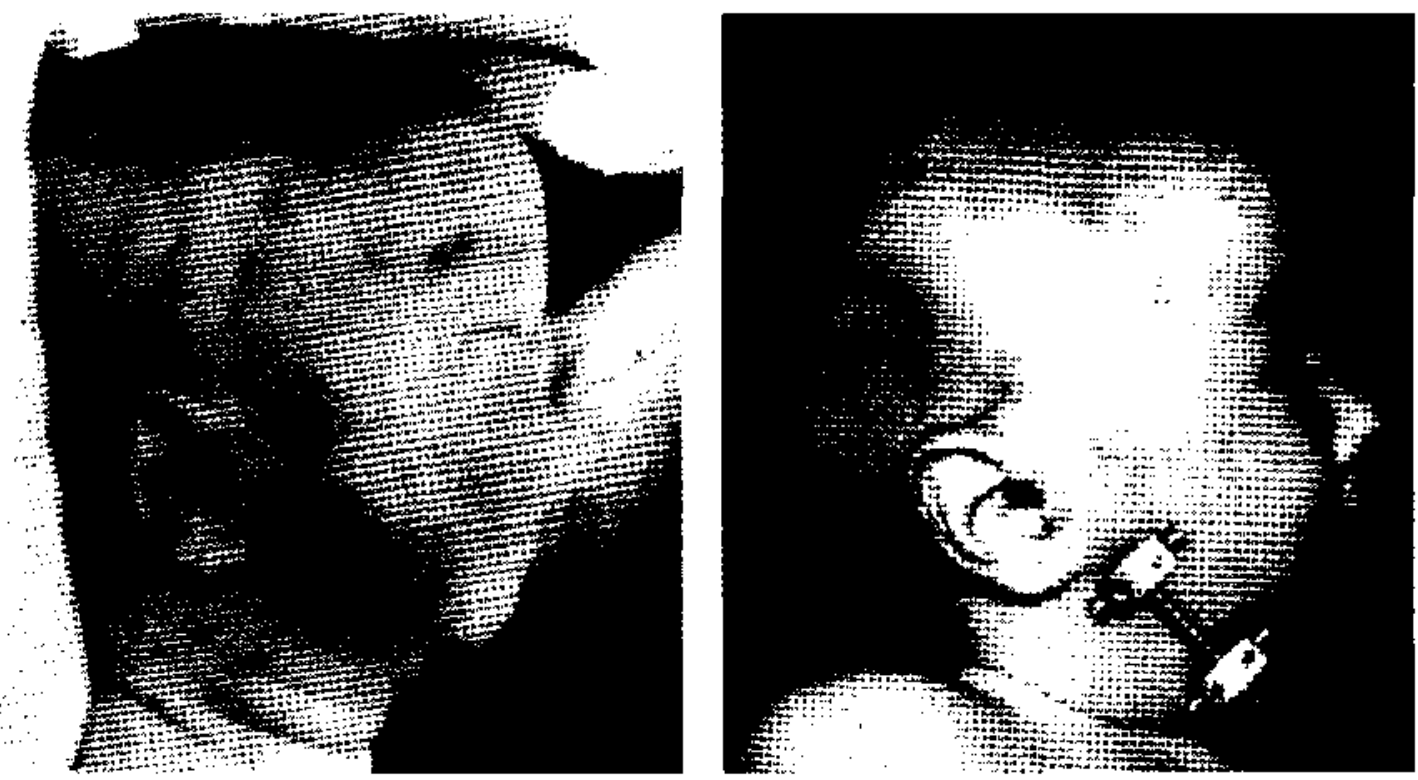

Figura 2: Paciente 2. Izquierda: síndrome de Pierre Rohin, gran hipoplasia mandibular, obstrucción respiratoria, asistencia respiratoria mecánica, por lo que se debe mantencr conectado a ventilación mecánica dada su obstrucción respiratoria. Derecha: durante el proceso di tratción mandibular usando el tractor externo tipo Molina, el avance nandibulas es cvidente y se ha retirado la asistencia respiratoria mecánica. 


\section{Resumen}

El alargamiento 6seo mediante tracción gradual es una nueva herramienta para el tratamiento de las malfonnaciones óseas del esqueleto facial. Utilizamos esta técnica en dos pacientes con síndrome de Pierre Robin portadores de hipoplasia mandibular severa con obstrucción respiratoria crítica, logrando un alargamiento mandibular importante (entre $1,6 \mathrm{~cm}$ y $2,2 \mathrm{~cm}$ ), suficiente para per mitir el cierre de la traqueostomía en un paciente y retirar de asistencia respiratoria mecánica al segundo caso. Con este método relativamente sencillo se logran excelentes resultados estético y funcional con muy pocos inconvenientes.

(Palabras clave: síndrome de Pierre Robin, hipoplasia de maxilar inferior, dificultad respiratoria, estiramiento óseo, tracción ósea gradual.)

\section{Referencias}

1. Codivilla A: On the neans of lengthening in the lower limb, the muscles and tissues which are shortened through deformity. Am J Orthop Surg 1905; 2: 353-369.

2. Lizarov $G$, Lediov $V$. Shitin $V$; The course of compact bone repartive regeneration in distraction osteosynthesis under different conditions of bone fragment fixation and experimental study (Russian). Exp Khir Anesteziol 1969; 14:3-12.

3. Nizarov G, Deviatov A. Trokhov V: Surgical lengthening of the shortened lower extremities (Russian). Vestin Khir 1972: 107: 100-103.

4. Snyder C. Levine G. Swanson H. Browne E: Mardibular lengthening by gradual distraction: preliminary report. Plast Reconstr Surg 1973; 51 : 506-508.

5. Karp N. Thorne C. Mc Carthy J. Sisson H: Bone lengthening in the craniofacial skeleton. Ann Plast Surg 1990; 24:231-237.

6. Karp N. Mc Carthy J. Schreiber J. Sisson H: Thorne CHM. Membranous bone lengthening: a serial histological study. Ann Plast Surg 1992; 29: 2-7.

7. Mc Carthy J. Schreiber J, Karp N. Thorne C, Grayson B. Lengthening the human mandible by gradual distraction. Plast Reconstr Surg 1992; 89: 1-8.

8. $M C$ Carthy $J$ : The role of distraction osteogenesis in the reconsiruction of the mandible in unilateral craniofacial microsomia. Clinics Plast Surg 1994; 2l: 625-63].

9. Delorme $R$, Larocaue $Y$, Caunette-Laberge L: Innovative surgical approach for the Pierre Robin avomalad: Subperiosteal release of the floor of the mouth musculature. Plast Reconstr Surg 1989; 83: $960-966$.

\section{AVISO A LOS AUTORES}

Con el objeto de acelerar la edición de los trabajos se solicita encarecidamente a los autores que disponen de computador con procesadores de texto IBM compatibles, enviar -en lo posible junto con las dos copias impresas de su material-, otra en disco flexible (tamaño 3,5" o 5,25", de doble o alta densidad) del mismo texto. El disco será devuelto de inmediato por correo certificado o por mano al autor corresponsal. 